\title{
Control of Demagnetizing Field and Magnetostatic Coupling in FeCoV Wires for Zero-Speed Sensor
}

\author{
Yasushi Takemura $^{1}$, Takamasa Aoki ${ }^{1}$, Hiroyuki Tanaka ${ }^{1}$, Tsutomu Yamada ${ }^{1}$, Susumu Abe $^{2}$, Shiro Kohno ${ }^{3}$, and \\ Hiroshi Nakamura ${ }^{3}$ \\ ${ }^{1}$ Department of Electrical and Computer Engineering, Yokohama National University, Yokohama 240-8501, Japan \\ ${ }^{2}$ Kanagawa University, Yokohama 221-8686, Japan \\ ${ }^{3}$ Nikkoshi Co., Ltd., Tokyo 103-0023, Japan
}

\begin{abstract}
A fast magnetization reversal in twisted Vicalloy wires is utilized in speed sensor, rotation sensor, and other applications. It has the advantage of generating a constant pulse voltage in a pickup coil which is independent of a changing rate of the applied magnetic field. But the demagnetizing field and magnetostatic coupling at both ends of the wire prevent desirable conditions of excitation magnets and symmetric output voltage in the sensor application. We have achieved improvements in the output voltage and the condition of the exciting magnetic field by using the twisted Vicalloy wire whose outer layer at both ends is etched.
\end{abstract}

Index Terms-Large Barkhausen jump, magnetic sensor, magnetic wire.

\section{INTRODUCTION}

A MAGNETIZATION reversal in magnetic wires with bistable magnetization states induces a pulse voltage in a pickup coil [1], [2]. The amplitude of the voltage does not depend on a frequency of the applied magnetic field down to zero. It is because that the magnetization switching is accompanied with a large Barkhausen jump. A twisted Vicalloy $(\mathrm{FeCoV})$ wire has been utilized in various applications of rotation sensor, speed sensor, and others as the optimum material yielding this phenomenon known as the Wiegand effect [1]. The twisted Vicalloy wire reveals a uniaxial magnetic anisotropy along the wire direction. Its coercive force is about $20 \mathrm{Oe}$ in the outer shell (soft layer) and 60 Oe in the inner core (hard core), respectively. When the magnetization of the soft layer is switched by an external magnetic field, a pulse voltage is induced in a pickup coil. Normally, the magnetization of the hard core is not reversed in the sensor application. Owing to the asymmetric magnetic structure of the soft layer and the hard core, the switching magnetic fields for realizing the parallel and antiparallel alignments in their magnetization states are not equivalent. The output voltages obtained during the parallel and antiparallel magnetization processes in a pickup coil are also not equivalent. These asymmetric properties in the exciting magnetic field and the output voltage are not preferable for the sensor application. In this paper, the control of the demagnetizing field and the magnetostatic coupling in the twisted Vicalloy wire have been studied in order to improve its sensor performance.

\section{EXPERIMENTS}

A semihard magnetic Vicalloy wire $(40 \mathrm{Fe}-50 \mathrm{Co}-10 \mathrm{~V})$ of $0.25 \mathrm{~mm}$ in diameter was used in this study. The double layered magnetic structure was achieved by twisting the wire [3]. Fig. 1(a) and (b) shows schematics of magnetization alignments

Digital Object Identifier 10.1109/TMAG.2006.879751

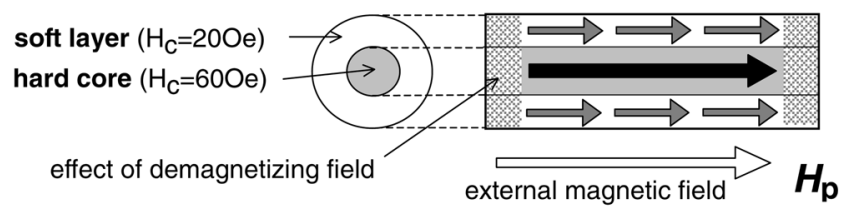

(a)

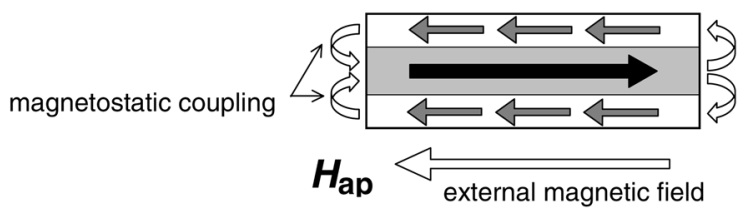

(b)

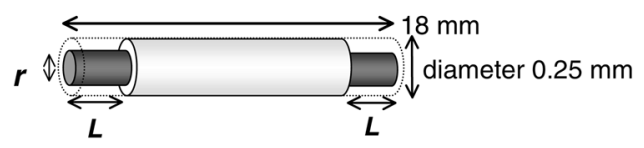

(c)

Fig. 1. Schematics of magnetic structures of the twisted Vicalloy wire under (a) parallel and (b) antiparallel alignments of a soft layer and hard core. (c) The outer layer at both ends of the as-prepared wire was etched in order to reduce effects of demagnetizing field and magnetostatic coupling.

of the twisted wire under the parallel and antiparallel configurations, respectively. When the magnetization of the soft layer is switched parallel to that of the hard core by the external magnetic field of $H_{\mathrm{p}}$, a pulse voltage is induced in a pickup coil due to a large Barkhausen jump. In the antiparallel switching process by the applied field of $H_{\text {ap }}$, the amplitude of the induced voltage is quite lower, which is consistent with the gradual reversal of the magnetization curve of the wire. The solid circles in Fig. 2 show the minor $B-H$ curve of the twisted wire measured during the magnetization reversal of the soft layer. The magnetization of the hard core was kept constant. The positive and negative directions of the applied field in the figure identified with the direction of $H_{\mathrm{p}}$ and $H_{\mathrm{ap}}$, respectively. The magnetization switching to the parallel alignment is steep, whereas that to the antiparallel alignment is gradual. Because of this switching feature, the wire has the disadvantage of the asymmetric output of 


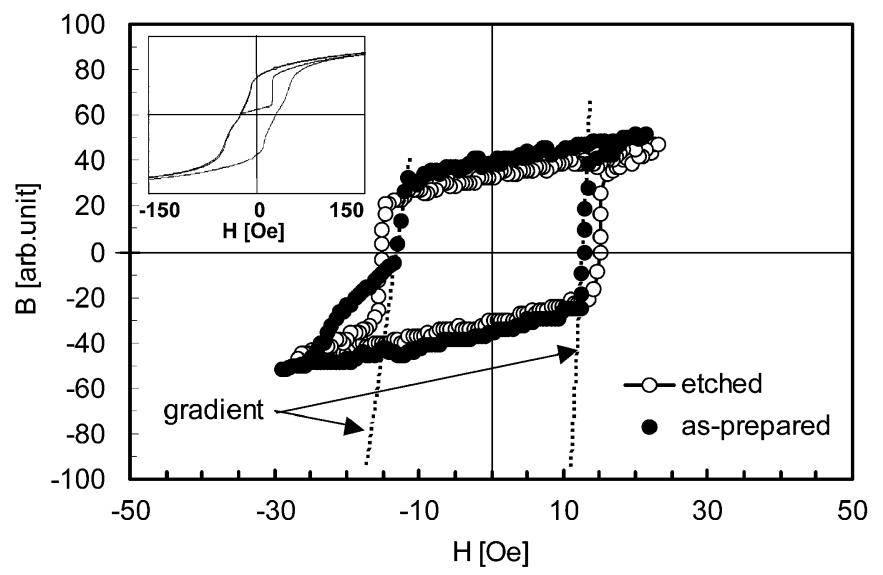

Fig. 2. $B-H$ loops of the as-prepared and etched Vicalloy wires. The dotted lines indicate gradients of the curves corresponding to the magnetization reversal of the soft layer. The insertion indicates the minor loop and the $B-H$ loop at a higher field.

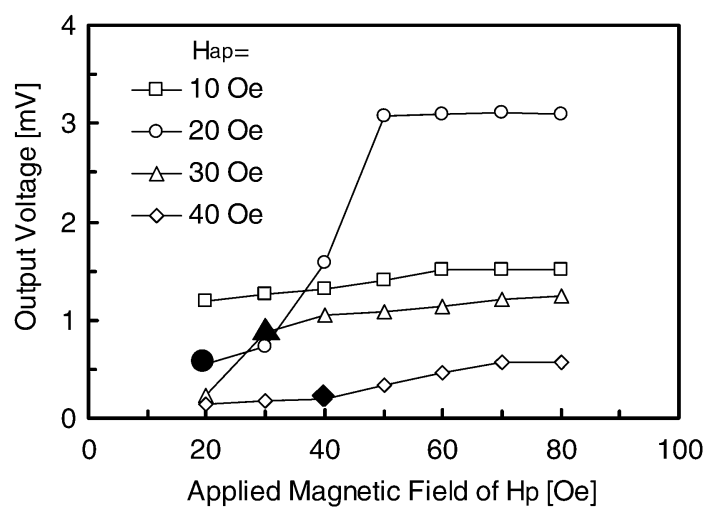

Fig. 3. Output voltage per one turn of a pickup coil obtained from the as-prepared Vicalloy wire. Asymmetric alternating magnetic field consisting of various combinations of $H_{p}$ and $H_{\text {ap }}$ at $70 \mathrm{~Hz}$ was applied to the wire.

the positive/negative voltages generated in the parallel/antiparallel magnetization processes, respectively.

Another disadvantage arises from a demagnetizing field in the parallel alignment [3], and also from a magnetostatic coupling in the antiparallel alignment [4] as illustrated in Figs. 1(a) and (b), respectively. The amplitude of the pulse voltage induced in a pickup coil (3000 turn, $10 \mathrm{~mm}$ length) was measured by applying an asymmetric alternating magnetic field at $70 \mathrm{~Hz}$, which consisted of various combinations of field strength for $H_{\mathrm{p}}$ and $H_{\text {ap }}$. Fig. 3 shows the output voltage obtained during the parallel switching process. The peak voltage per one turn of the pickup coil is used to quantify the induced pulse voltage. The length of the wire was $30 \mathrm{~mm}$ in this measurement. The higher voltage was obtained by the applied field of $H_{\mathrm{p}}>50$ Oe and $H_{\mathrm{ap}}=20$ Oe. In case of the larger applied filed of $H_{\mathrm{ap}}>$ $30 \mathrm{Oe}$, the output voltage was decreased because of the partial magnetization reversal of the hard core. It can be understood by the $B-H$ loop at a larger field and the minor loop inserted in Fig. 2. The output voltage obtained by the symmetric applied field of $H_{\mathrm{p}}=H_{\mathrm{ap}}$ is less than $1 \mathrm{mV}$ as indicated by the solid symbols in the figure. This lower voltage under the symmetric exciting field is not desirable for sensor applications. In order to improve these output properties by reducing the effects of the

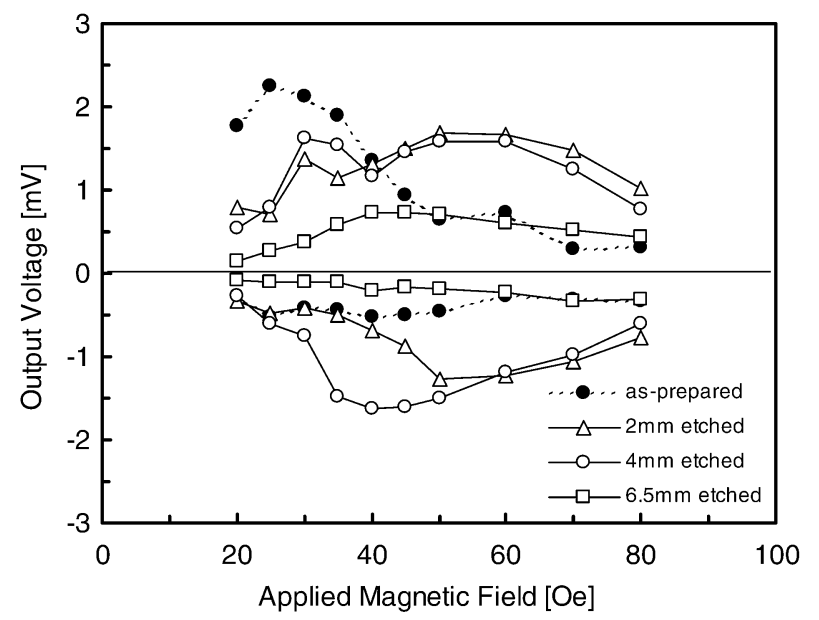

Fig. 4. Output voltage per one turn of a pickup coil obtained from the as-prepared and etched Vicalloy wires. Symmetric alternating magnetic field at $70 \mathrm{~Hz}$ was applied to the wires.

demagnetizing field and the magnetostatic coupling in the wire, the outer layer (soft layer) at both ends was etched by a $\mathrm{FeCl}_{2}$ solution as shown in Fig. 1(c).

\section{RESULTS AND DISCUSSION}

The output voltages generated from the as-prepared (twisted) and etched wires were compared. The pulse voltage was measured by applying a symmetric alternating magnetic field at 70 $\mathrm{Hz}$ to the wires. The length of the as-prepared wire was $18 \mathrm{~mm}$. The length of the etched part from each end of the wire, indicated by $L$ in Fig. 1(c), was 2, 4, or $6.5 \mathrm{~mm}$. The diameter of the etched part was reduced to $r=0.15 \mathrm{~mm}$. Fig. 4 shows the positive and negative output voltages obtained during the parallel and antiparallel switching processes, respectively. As previously described, the asymmetric positive/negative output voltage was observed from the as-prepared wire. For a lower exciting field below $40 \mathrm{Oe}$, the positive output is relatively large, which is a typical phenomenon of the large Barkhausen jump. But the negative output is lower. It is also disadvantageous that the positive output is reduced for a higher exciting field. This reduced voltage was attributed to the magnetization reversal of the hard core. The output properties were improved in the etched wires. The wires with their etched length $L=2$ and $4 \mathrm{~mm}$ exhibited the symmetric positive/negative output voltage. The output voltage was not so reduced in the higher exciting field.

Considering that a demagnetizing field factor is determined by the aspect ratio of length/diameter of the wire, the demagnetizing field for the soft layer in the etched wire is reduced. It is because that the soft layer is isolated from the hard core and that the aspect ratio of the soft layer determined by its length/layer thickness is increased. When positions of the both ends of the soft layer and hard core are different, their magnetostatic coupling is reduced. As for the wire of $L=6.5 \mathrm{~mm}$, the output voltage was quite low due to the small volume of the remaining soft layer. Its asymmetric output is presumably attributed to a higher demagnetizing field due to a short length $(5 \mathrm{~mm})$ of the soft layer.

It was found that the both of positive and negative output voltages were independent of the excitation frequency in the etched 


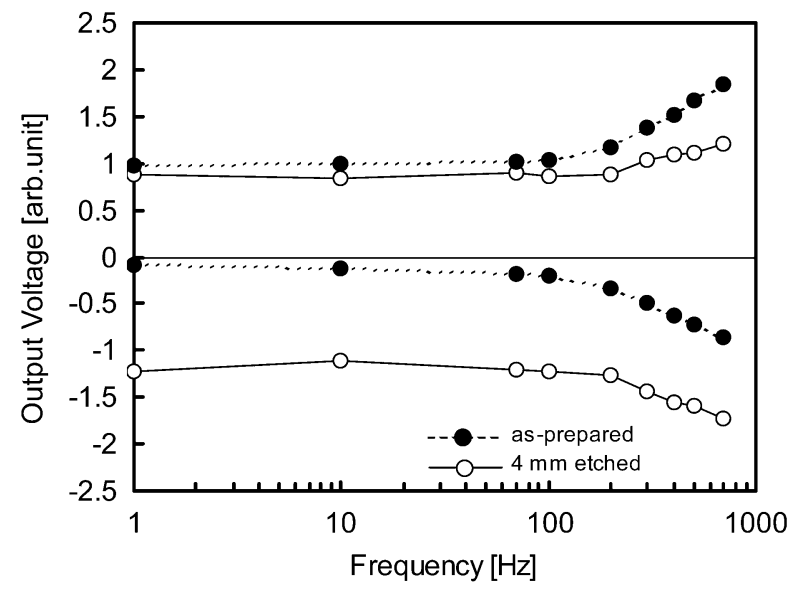

Fig. 5. Excitation frequency dependence of output voltage from the as-prepared and etched Vicalloy wires. Symmetric alternating magnetic field of 40 Oe was applied to the wires.

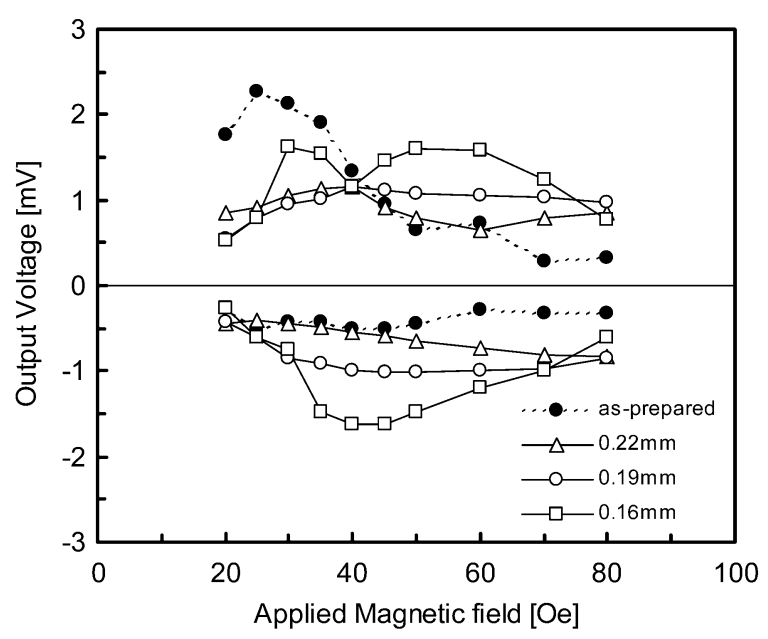

Fig. 6. Output voltage per one turn of a pickup coil obtained from the as-prepared and etched Vicalloy wires. Symmetric alternating magnetic field at $70 \mathrm{~Hz}$ was applied to the wires.

wire ( $L=4 \mathrm{~mm}$ ), which could not be achieved by the as-prepared wire as shown in Fig. 5. The symmetric alternating magnetic field of $40 \mathrm{Oe}$ at various frequency was applied. As the negative output voltage obtained in antiparallel magnetization process of the as-prepared wire is not accompanied with a large Barkhausen jump, its amplitude is small and depends on the excitation frequency. The output voltage was increased by the induced electromotive force that was superimposed onto the constant voltages above $100 \mathrm{~Hz}$.

The wires with different diameters of the etched part $r=$ $0.22,0.19,0.15 \mathrm{~mm}$ were prepared. The wire length was $18 \mathrm{~mm}$, and the etched length was constant at $L=4 \mathrm{~mm}$. Fig. 6 shows their output voltage measured by applying a symmetric alternating magnetic field at $70 \mathrm{~Hz}$. With decreasing the diameter of the etched part, the symmetry in the positive/negative output was improved. It is also obvious that the negative output voltage was increased by the etching. The largest voltage was obtained

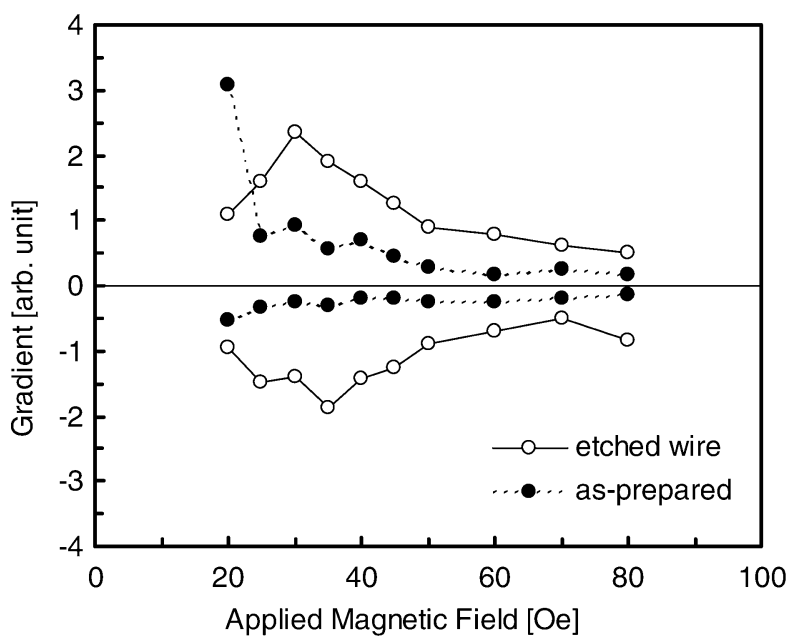

Fig. 7. Gradient of magnetization reversal of the soft layers in the as-prepared and etched Vicalloy wires calculated from their $B-H$ minor loops.

with the wire of $r=0.15 \mathrm{~mm}$. A sample with a thinner etched part was not prepared in terms of instability and damage.

Fig. 2 (open circles) shows the $B-H$ curve of the etched sample $(L=4 \mathrm{~mm}, r=0.15 \mathrm{~mm})$. The curve represents the minor loop corresponding to the magnetization switching of the soft layer. The as-prepared wire exhibited the asymmetric parallel/antiparallel switching of the soft layer against the hard core, while the etched wire exhibited almost symmetric. The gradient of the magnetization reversal was plotted as a function of the maximum applied field $\left(H_{\mathrm{p}}=H_{\mathrm{ap}}\right)$ in measuring the $B-H$ curves as shown in Fig. 7. The gradients for the switching to the parallel and antiparallel alignments are indicated by the positive and negative directions, respectively. As the amplitude of the induced pulse voltage is determined by a changing rate of the magnetic flux during the magnetization reversal, the improved output properties of the etched wires agrees with the gradients of the magnetization switching.

In summary, the symmetric positive/negative output voltage was obtained in the etched Vicalloy wires. As the output voltage is independent of frequency of the exciting magnetic field down to zero, this improvement is useful for sensor applications including zero-speed sensor. A condition of the exciting magnetic field in its polarity and field strength is also improved, which allows to use pairs of permanent magnets efficiently and extends a range of the magnet/wire distance in applications.

\section{REFERENCES}

[1] J. R. Wiegand and M. Velinsky, U.S. Patent 3820 090, 1974.

[2] M. Vázquez, C. Gómez-Polo, D.-X. Chen, and A. Hernando, "Magnetic bistability of amorphous wires and sensor applications," IEEE Trans. Magn., vol. 30, no. 2, p. 907, Mar. 1994.

[3] A. Matsushita and Y. Takemura, "Power generating device using compound magnetic wire," J. Appl. Phys., vol. 87, no. 9, p. 6307, May 2000.

[4] Y. Takemura and A. Matsushita, "Frequency dependence of output voltage generated from bundled compound wires," IEEE Trans. Magn., vol. 37 , no. 4, p. 2862 , Jul. 2001.

Manuscript received March 13, 2006 (e-mail: takemura@ynu.ac.jp). 